\title{
An Analysis on the Effects of Environmental Disaster using Pooled Disjoint Block Triangular Fuzzy Cognitive Maps
}

\author{
Nivetha Martin* \\ Assistant Professor, Department of Mathematics, Arul Anandar College (Autonomous), Karumathur - 625514, \\ Tamil Nadu, India; nivetha.martin710@gmail.com
}

\begin{abstract}
Nature is a gift of god to man who enjoys its benefits to great extent without resource restoration. This act of human has caused environmental degradation which has now resulted in environmental disasters such as climate change, loss of biological diversity, desertification, pollution, ozone depletion and accumulation of organic pollutants. The effects of these catastrophes on the surroundings make it unfit for the living beings to survive. People are not aware of the real effects of such calamities to the fullest, if there is deficit of any resources they instantly opt for alternatives without taking any steps for refurbishment. This attitude has to be changed by making them to understand the consequences of environmental disaster are not to be negligible but to be cautious for which Pooled Disjoint Fuzzy Cognitive maps is used. As this method was applied for several analyses earlier, it has now been modified with the inclusion of Triangular- fuzzy numbers to deal the precision variables. In this method the impact is quantified not with numerical values but with linguistic variables so as to determine the effects of environmental disaster in a most profound manner. This method indeed finds the impacts of the effects over each other and their interrelation so as to formulate preventive steps for it at the earliest to create green world.
\end{abstract}

Keywords: Environmental Disaster, Linguistic Variables, Pooled Disjoint Block Fuzzy Cognitive Maps, Triangular Fuzzy Numbers

\section{Introduction}

Environmental disaster has now emerged as a serious crisis. Disturbance of the nature ends in horrified effects which ruins the entire framework of the eco system. Natural calamities are predicted but not averted. The modern technology alarms the people to protect themselves from these natural calamities whereas environmental disasters are man -made misfortunes whose occurrences are witnessed every moment in these days, but they are not cared much. The effect of one over another is cyclic as all are interdependent and interrelated. The reflection of these effects is highly miserable as it paves way for resource depletion. The impacts of environmental disaster are considered as concepts, to analyze the inter- reliance the concept of fuzzy Cognitive maps is exercised which is the successor of cognitive maps.
Cognitive Maps, which are precursors of Fuzzy Cognitive Maps, were first introduced in 1976 by a political scientist named Robert Axelrod (Axelrod, 1976). They were presented as a tool to help analyze systems that include concepts interrelated by complex relationships. In particular, Cognitive Maps were applied to represent social scientific knowledge. A Cognitive Map may be expressed in a simple digraph form consisting of nodes and edges whereby each node corresponds to a concept or variable relevant to a domain of application. This set of nodes is connected by directed edges that represent mutual relationships among concepts. If the relationship between the concepts influences each other positively then the edge is assigned positive weightage and negative value is assigned between the edges if the concepts do not influence each other. If there is no edge between the given two concepts, it indicates that there is no direct

${ }^{*}$ Author for correspondence 
cause-effect correlation linking these concepts. However, the results of this modeling technique turned out to be insufficient to describe more complex systems due to the limited representation of relations. Typically, causality is not Boolean (two- valued, yes-no) in real-life systems, i.e., the relationships are too complex to be described with only a sign. This was the motivation to extend the theory of Cognitive Maps.

Ten years later, in 1986, Kosko (Kosko, 1986) introduced Fuzzy Cognitive Maps (FCMs). The fundamentals of this method, in terms of model representation as a set of concepts connected by relationships, are the same as in the Axelrod's approach. In comparison to Cognitive Maps, the most significant generalization of FCMs lies in the manner the contributory associations between concepts are represented. Instead of using just a sign, the contributory association is given a value which represents the intensity of the association. FCMs describe relationships in fuzzy terms, such as highly low, medium, strong, or highly strong. In other words, a weight associated with directed edge from a node $A$ to node $B$ quantifies- how much concept A causes B. The strength of a relationship between two nodes (i.e., weight value) is usually normalized on the closed interval -1 to 1 . The value -1 represents maximum negative, whereas the value +1 , maximum positive influence. Zero denotes no causal effect. Other values correspond to intermediate levels of influence. Therefore, FCM is totally represented by concepts and its association. Later many methods such as induced fuzzy cognitive maps, induced linked fuzzy cognitive maps, Topsis, consensus centrality measures, multi step fuzzy cognitive maps, simple and weighted FCM, Pooled Disjoint Block Fuzzy Cognitive Maps.

These methods are modified in accordance to the situation. In this paper, Pooled Disjoint Block Fuzzy Cognitive Maps method is modified into Pooled Disjoint Block Triangular Fuzzy Cognitive Maps method which ranks the attributes by assigning weightage.

The structure of the article is as follows: Section 2 contains the elementaries Section 3 presents the methodology, Section 4 briefs out the effects of environmental disaster, Section 5 makes inferences of the results and Section 6 brings the paper to close.

\section{Triangular FCM (TFrFCM)}

A TFrFCM is a directed graph with concepts represented by fuzzy sets as nodes and edges representing the causal relationships between the concepts with certain weights assigned. A TFrFCM is cyclic if it has a feedback and the system becomes dynamic and attains equilibrium if so the state vector becomes the fixed point. Several authors have given various definitions regarding the preliminaries of TFrFCM.

\subsection{Pooled Block Disjoint Fuzzy Cognitive Maps (PDFCM)}

The nodes if many in number, are grouped into disjoint classes and their interrelationship is studied. The FCM obtained for each class is aggregated to form a pooled block disjoint fuzzy cognitive map.

\subsection{Pooled Block Disjoint Triangular Fuzzy Cognitive Map (PDTFrFCM)}

The nodes in CBDFCM if represented as triangular fuzzy sets become Pooled Block Disjoint Triangular Fuzzy Cognitive Maps.

\section{Methodology}

Let TFrC1, TFrC2, ...TFrCn be the nodes of PDTFrFCM which are grouped into disjoint class and the FCM is first obtained. Then the pooled inter reliance is obtained from the experts. Once the connection matrix is formed, the procedure of pooled block disjoint is applied by merging the steps of TFrFCM. One of the concepts is kept in ON position and other states are kept in OFF position and it is passed into the connection matrix. TFrsum is obtained and TFrMaximum weight is found, to which the threshold operation is applied. It is the way of assigning 0 to the negative values and 1 to the positive values. The process is repeated until the fixed point is attained. This method is indeed very compatible and yield systematic results.

\section{Effects of the Environmental Disaster}

The effects of the environmental disaster are taken as triangular fuzzy sets and $m$ disjoint classes are formed. The causal relationships are obtained from the experts by the method of questionnaire. The below listed are considered as prime nodes:

TFrC1 - Habitat conversion and destruction 


\begin{tabular}{|c|c|c|}
\hline TFrC2 & \multicolumn{2}{|c|}{ - Degradation of the eco system } \\
\hline TFrC3 & \multicolumn{2}{|c|}{ Exploitation of Species } \\
\hline TFrC4 & \multicolumn{2}{|c|}{ Pollution } \\
\hline TFrC5 & \multicolumn{2}{|c|}{ Soil erosion } \\
\hline TFrC6 & \multicolumn{2}{|c|}{ carbon emission } \\
\hline TFrC7 & \multicolumn{2}{|c|}{ - Declining agricultural yield } \\
\hline TFrC8 & \multicolumn{2}{|c|}{ - loss of soil fertility } \\
\hline TFrC9 & \multicolumn{2}{|c|}{ - Potential reduction in food exports } \\
\hline TFrC10 & \multicolumn{2}{|c|}{$\begin{array}{l}\text { potential suppression of human immune } \\
\text { system }\end{array}$} \\
\hline TFrC11 & \multicolumn{2}{|c|}{$\begin{array}{l}\text { accelerated forms of allergies, disorders in } \\
\text { human health }\end{array}$} \\
\hline TFrC12 & \multicolumn{2}{|c|}{ - land degradation } \\
\hline $\mathrm{TFrC13}$ & \multicolumn{2}{|c|}{ depletion of stratospheric ozone } \\
\hline TFrC14 & \multicolumn{2}{|c|}{ emission of UV rays } \\
\hline TFrC15 & \multicolumn{2}{|c|}{ introduction of alternate products } \\
\hline \multicolumn{3}{|c|}{ The linguistic values of the Triangular Fuzzy Number are } \\
\hline \multicolumn{2}{|c|}{ very low $(v l)$} & $(0,0,0.250)$ \\
\hline \multicolumn{2}{|c|}{ low $(l)$} & $(0,0.250,0.50)$ \\
\hline \multicolumn{2}{|c|}{ medium $(m)$} & $(0.250,0.50,0.750)$ \\
\hline \multicolumn{2}{|c|}{ high $(h)$} & $(0.50,0.750,1)$ \\
\hline \multicolumn{2}{|c|}{ very high (vh) } & $(0.750,1,1)$ \\
\hline
\end{tabular}

The concepts are divided into 3 mutually disjoint classes as follows:

C1: ( TFrC1, TFrC2, TFrC3)

C2(TFrC5, TFrC7, TFrC8, TFrC9, TFrC12, TFrC15)

C3: (TFrC4, TFrC6, TFrC10, TFrC11, TFrC13, TFrC14)

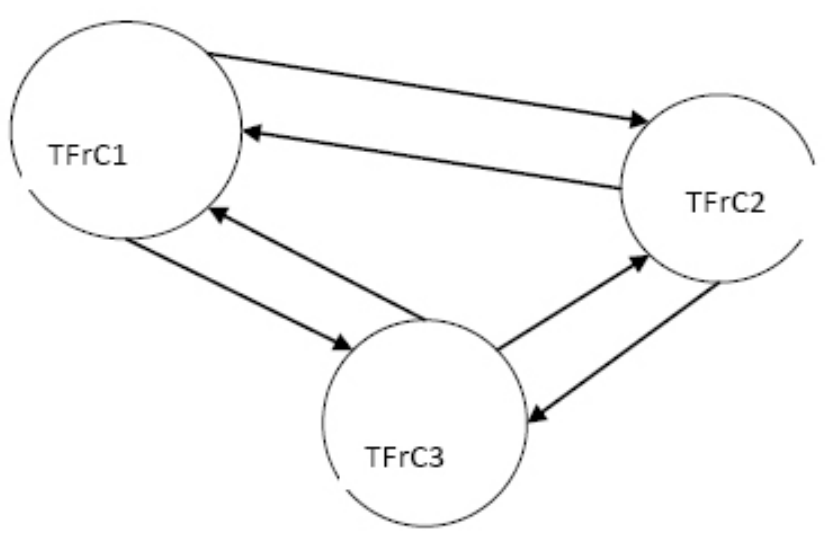

$\begin{array}{cccc} & \text { TFrC1 } & \text { TFrC2 } & \text { TFrC3 } \\ \text { TFrC1 } & 0 & L & h \\ \text { TFrC2 } & v h & 0 & h \\ \text { TFrC3 } & h & M & 0\end{array}$

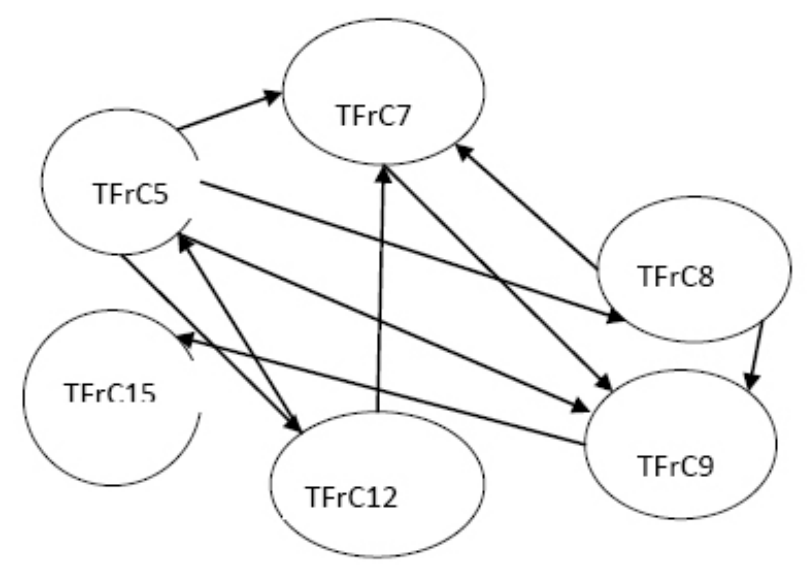

TFrC5 $\quad$ TFrC7 $\quad$ TFrC8 $\quad$ TFrC9 $\quad$ TFrC12 TFrC15

$\begin{array}{ccccccc}\text { TFrC5 } & 0 & V h & v h & v h & h & h \\ \text { TFrC7 } & v l & 0 & v l & h & v l & v l \\ \text { TFrC8 } & v l & H & 0 & v h & v l & v l \\ \text { TFrC9 } & v l & V l & v l & 0 & v l & h \\ \text { TFrC12 } & h & H & v l & v l & 0 & v l \\ \text { TFrC15 } & v l & v l & v l & v l & v l & 0\end{array}$

As all the concepts of the classes are related either directly or indirectly, the causal relationships are represented by linguistic values. The indirect impacts (which are not represented by edges) are assigned VL and self implication is assigned as zero.

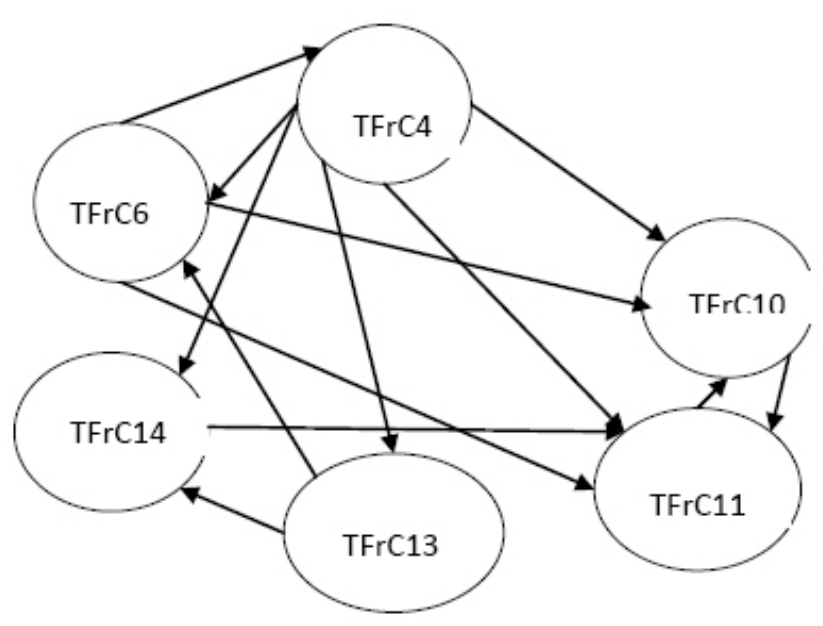


TFrC4 TFrC6 TFrC10 TFrC11 TFrC13 TFrC14

$\begin{array}{lllllll}\text { TFrC4 } & 0 & V h & v h & v h & H & v h\end{array}$

$\begin{array}{lllllll}\text { TFrC6 } & \text { vh } & 0 & h & h & \mathrm{Vl} & \mathrm{v} l\end{array}$

$\begin{array}{lllllll}\mathrm{TFC} C 10 & v l & V l & 0 & v h & V l & v l\end{array}$

$\begin{array}{lllllll}\text { TFrC11 } & 0 & 0 & m & 0 & 0 & 0\end{array}$

$\begin{array}{lllllll}\operatorname{TFrC13} & l & H & l & l & 0 & h\end{array}$

$\begin{array}{lllllll}\text { TFrC14 } & l & L & l & h & L & 0\end{array}$

In this FCM the concept TFrC11 has no impact on TFrC4, TFrC6, TFrC13, TFrC14 either directly or indirectly and therefore the value 0 is assigned. The Connection Matrix M of the Pooled Disjoint Block is given below:

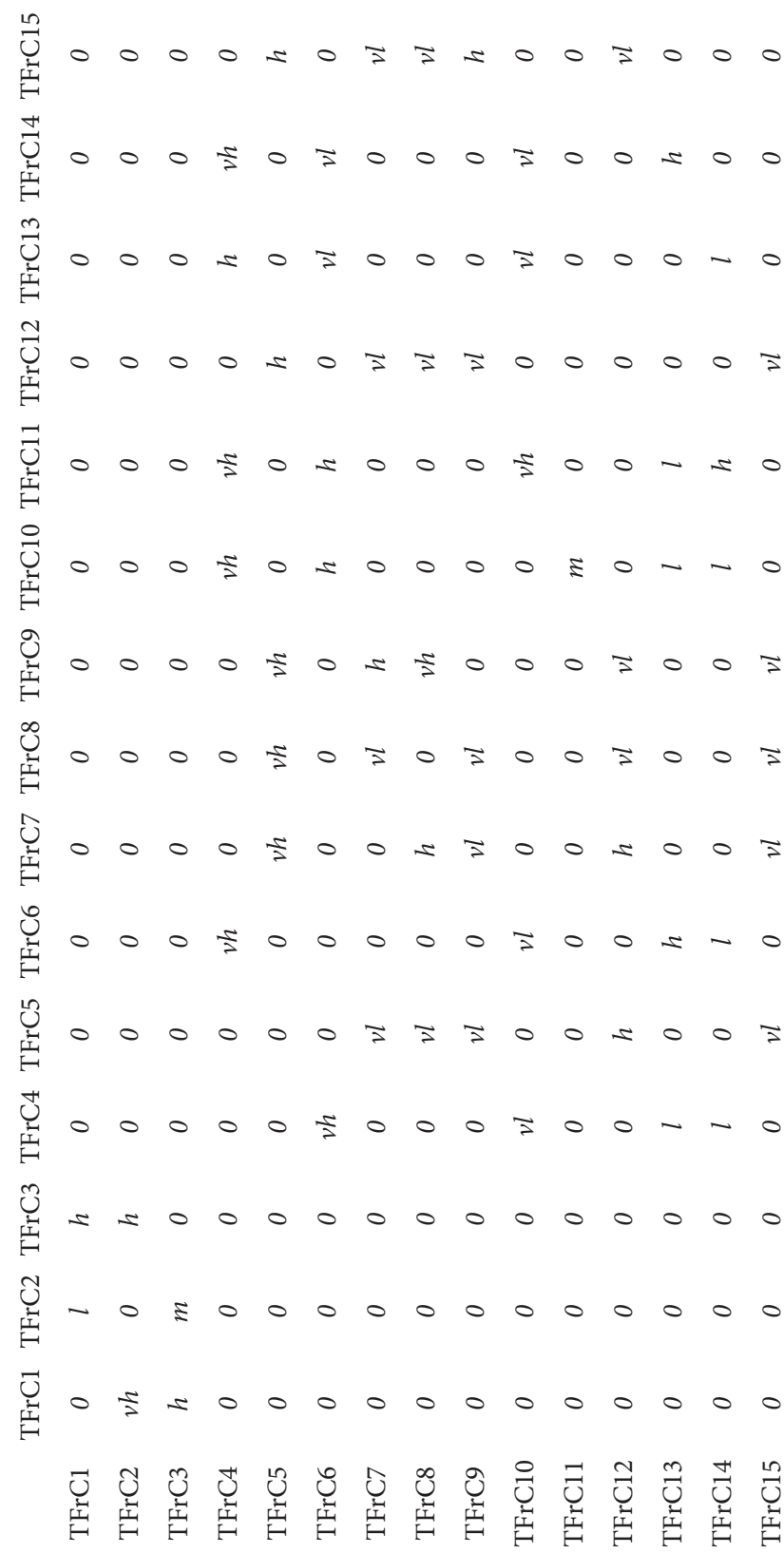

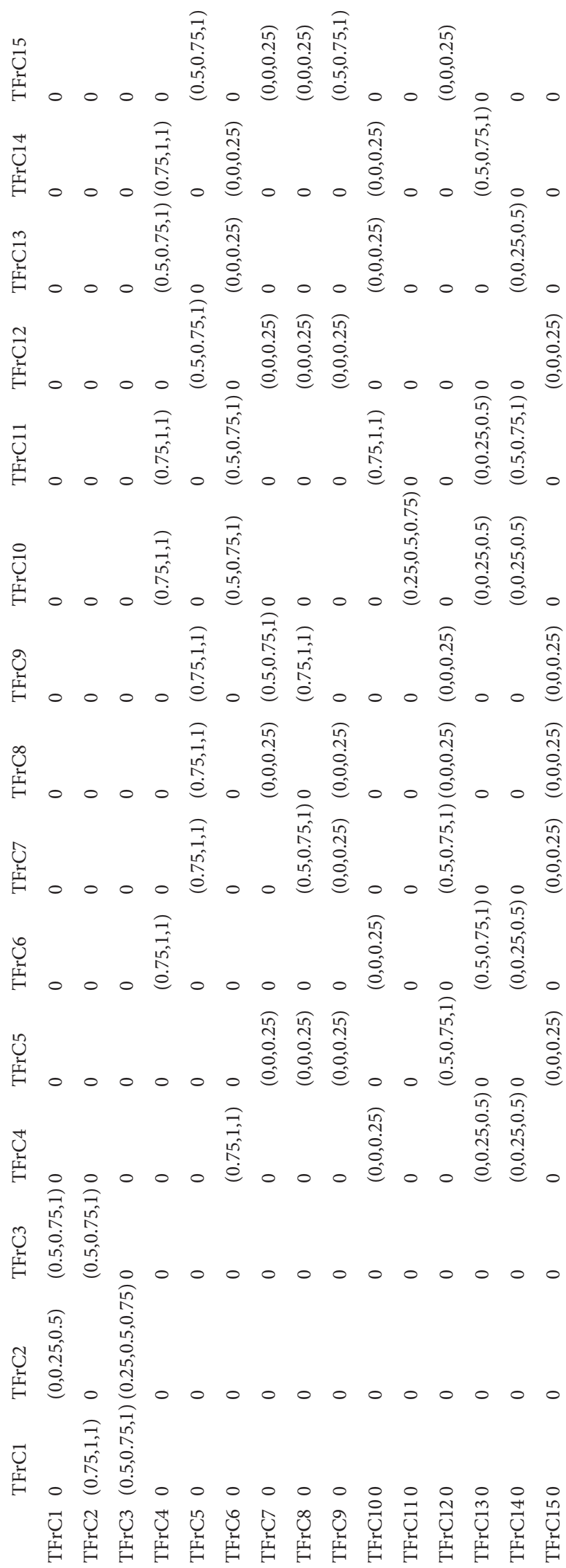


Consider each concept in $\mathrm{ON}$ position. Using the above methodology, the following triggering pattern is obtained:

\begin{tabular}{|c|c|}
\hline $\begin{array}{c}\text { ON POSITION OF THE } \\
\text { CONCEPT }\end{array}$ & TRIGGERING PATTERN \\
\hline$(100000000000000)$ & TFrC1 $\longrightarrow$ TFrC3 \\
\hline$(010000000000000)$ & TFrC2 $\longrightarrow$ TFrC3 \\
\hline$(001000000000000)$ & TFrC3 $\longrightarrow$ TFrC1 \\
\hline$(000100000000000)$ & TFrC4 $\longrightarrow$ TFrC6 \\
\hline$(000010000000000)$ & TFrC5 $\longrightarrow$ TFrC15 \\
\hline$(000001000000000)$ & TFrC6 $\longrightarrow$ TFrC4 \\
\hline$(000000100000000)$ & TFrC7 $\longrightarrow$ TFrC9 \\
\hline$(000000010000000)$ & TFrC8 $\longrightarrow$ TFrC9 \\
\hline$(000000001000000)$ & TFrC9 $\longrightarrow$ TFrC15 \\
\hline$(000000000100000)$ & TFrC10 $\longrightarrow$ TFrC11 \\
\hline$(000000000010000)$ & TFrC11 $\longrightarrow$ TFrC10 \\
\hline$(000000000001000)$ & TFrC12 $\longrightarrow$ TFrC7 \\
\hline$(000000000000100)$ & TFrC13 $\longrightarrow$ TFrC6 \\
\hline$(000000000000010)$ & TFrC14 $\longrightarrow$ TFrC10 \\
\hline$(000000000000001)$ & TFrC15 $\longrightarrow$ TFrC9 \\
\hline
\end{tabular}

\section{Discussion}

The triggering pattern clearly shows the high impact of these effects over another and it is also observed that the concepts TFrC3, TFrC9, TFrC10 and TFrC15 seems to be influenced by other concepts. Therefore, the repeated pattern clearly explicates that Exploitation of Species, Potential reduction in food exports, Potential suppression of human immune system and introduction of alternate products are the major impacts of environmental disaster which has to be concentrated.

\section{Conclusion}

This paper discusses emerge of environmental disaster and its effects in brief. The mathematical method used in this paper assist to determine the core impacts of these effects over another. It is indeed a step to integrate the aspects of mathematics with that of the environmental factors. By determining the prime impacts preventive and mitigation steps can be formulated so as to formulate precautious initiatives to promote greener world. The present world gets destroyed by every unit of time. This can't be averted immediately but controlled by repeated implementation of remedial measures for which this article aims.

\section{References}

1. Toffel MW. End-of-life product recovery: Prior research and future directions [Working paper]. Berkely: Haas School of Business, University of California; 2002. Available from: http://www.toffel.com

2. Ferguson ME, Toktay LB. The effect of competition on recovery strategies. Production and Operations Management. 2006; 15(3):351-68.

3. Kovacs G. Corporate environmental responsibility in the supply chain. Journal of Cleaner Production. 2008; 16(15):1571-8.

4. Sarkis J, Zhu QH, Lai KH. An organizational theoretic review of green supply chain management literature. International Journal of Production Economics. 2011; 130(1):1-15.

5. Vachon S, Klaseen RD. Supply chain management and environmental technologies: the role of integration. International Journal of Production Research. 2007; 45(2):401-23.

6. Chin MCA. Identifying communities in blogs: Roles for social network analysis and survey instruments. International Journal of Web Based Communities. 2007; 3(3):345-63.

7. Del Pozo M, Manuel C, González-Arangüena E, Owen G. Centrality in directed social networks. A game theoretic approach. Social Networks; 2011.

8. Freeman LC. Centrality in social networks conceptual clarification. Social Networks. 1978; 1(3):215-39.

9. Chen J, Saad Y. Finding dense subgraphs for sparse undirected, directed and bipartite graph. Minnesota: University of Minnesota Twin Cities; 2009.

10. Obiedat M, Sandhya S, Strickert G. A new method for identifying the central nodes in fuzzy cognitive maps using consensus centrality measure. 19th International Congress on Modeling and Simulation; Perth, Australia, 2011 Dec 12-16.p.1084-91.

11. Kosko B. Fuzzy associative memory systems, Kandel A, editor. Fuzzy Expert Systems. Boca Raton: CRC Press; 1992a. p. 135-62.

12. Kosko B. Neural networks and fuzzy systems: a dynamical systems approach to machine intelligence. Englewood Cliffs, NJ: Prentice-Hall; 1992b.

13. Herrera F, Martinez L. An approach for combining linguistic and numerical information based on the 2-tuple fuzzy linguistic representation model in decision-making. International Journal of Uncertainty Fuzziness and Knowledge-Based Systems. 2000b; 8(5):539-62. 
14. Delgado M, Verdegay JL, Vila MA. On aggregation operations of linguistic labels. International Journal of Intelligent Systems. 1993; 8(3):351-70. DOI: 10.1002/ int.4550080303.

15. Botafogo RA, Rivlin E, Shneiderman B. Structural-analysis of hypertexts identifying hierarchies and useful metrics. ACM Transactions on Information Systems. 1992; 10(2):142-80.

16. Kaligarani J, Martin N, Meenakshi M. Causes of math anxiety in engineering students-an analysis using Induced Fuzzy Cognitive Maps (IFCM) with TOPSIS. Global Journal of Pure and Applied Mathematics. 2015; 11(5):2705-18.
17. Martin N, Kaligarani J, Meenakshi M. An analysis of driving performance error using Fuzzy Triangular four matrix of success and new hexagonal fuzzy number in Fuzzy Cognitive maps. International Journal of Applied Engineering Research. 2015; 10(85):423-9.

18. Martin N, Gandhi NR, Pandiammal P. Bio-friendly EPQ inventory model incorporating the cost of green energy to create light pollution free society. International Journal of Applied Engineering Research. 2015; 10(85):430-8.

19. Martin N, Ritha W. Environmental oriented inventory model and benefits of incineration as waste disposal method. Ariyabhatta Journal of Mathematics. 2014; 5(1):159-64. 